\title{
A time series analysis of the population genetics of the self-incompatibility polymorphism. 2. Frequency-equivalent population and the number of alleles that can be maintained in a population
}

\author{
ROGER J. BROOKS $\uparrow$, ANDREW M. TOBIAS $\uparrow \&$ MICHAEL J. LAWRENCE $* *$ \\ †Operational Research Group, School of Manufacturing and Mechanical Engineering, University of Birmingham, \\ Birmingham B15 2TT and $\ddagger$ Wolfson Laboratory for Plant Molecular Biology, School of Biological Sciences, \\ University of Birmingham, Birmingham B15 2TT, U.K.
}

\begin{abstract}
The concept of a frequency-equivalent population is introduced, which is the panmictic population that has the same allele frequency distribution as a given population with overlapping generations and variation in plant size. It differs from the traditional concept of effective population size, $N_{\mathrm{e}}$, in that the effect of variation in plant size is to make the frequencyequivalent population larger than the actual population, whereas the effective population size is smaller. Overlapping generations has the opposite effect. The number of alleles that can be maintained in a population at equilibrium is calculated incorporating these two factors, with variation in plant size significantly reducing the number of alleles and overlapping generations increasing the number of alleles that can be maintained.
\end{abstract}

Keywords: effective population size, mathematical theory, population genetics, seed dormancy, self-incompatibility polymorphism, variation in plant size.

\section{Introduction}

A general expression for the probability distribution of the frequency of a self-incompatibility allele was derived in the previous paper (Brooks et al., 1997). The frequency, $f_{t}$, of an allele in the $t$ th flowering episode of a population of constant size $N$, containing $k$ alleles and two sizes of plant, and with overlapping generations, was given by:

$f_{t}=\frac{2 N}{k}+z_{t}+d \theta \sum_{i=1}^{t-1}(1-d+d \theta)^{i-1}\left(z_{t-i}+L z_{l, t-i}\right)$,

where $d=$ probability of a seed germinating in a particular flowering episode,

$L=\frac{l_{q}-1}{1+l_{p}\left(l_{q}-1\right)}$,

\section{${ }^{*}$ Correspondence.}

§Present address: IACR Long Ashton Research Station, Department of Agricultural Sciences, University of Bristol, Long Ashton, Bristol BS18 9AF, U.K. $l_{p}=$ proportion of large plants,

$l_{q}=$ ratio of the quantity of seed and pollen of the large plants compared to small plants,

and $\theta=1-\frac{k}{(k-1)(k-2)}$,

with initial conditions, $f_{t}=2 N / k$ for $t \leq 0$.

The $z$ s are normal random variables with a zero mean. The average variance of these random variables for any value of $t$ is approximately given by:

$\operatorname{var}\left(z_{t}\right)=\frac{2 N(k-2)}{k^{2}}$

$\operatorname{var}\left(z_{l, t}\right)=\frac{2 N l_{p}\left(1-l_{p}\right)}{k}$

For steady state, $t=\infty$, and so the allele frequency is normally distributed with a mean of $2 N / k$ and a variance given by: 


$$
\begin{aligned}
\operatorname{var}\left(f_{\infty}\right)= & \frac{2 N(k-2)}{k^{2}}\left(1+\frac{d^{2} \theta^{2}}{1-(1-d+d \theta)^{2}}\right) \\
& +\frac{2 N l_{p}\left(1-l_{p}\right)}{k} \frac{d^{2} \theta^{2} L^{2}}{1-(1-d+d \theta)^{2}} .
\end{aligned}
$$

\section{Frequency equivalent population}

Equation 2 gives the steady-state allele frequency distribution for a population with overlapping generations (seed dormancy, denoted D) and variation in plant size (the existence of large plants, denoted L). This model is denoted the DL model. The same equation also holds for a panmictic population (denoted the NIL model) but with $d=1$ and $l_{p}=$ either 0 or 1 . For a given DL model, a NIL model with the same allele frequency distribution, referred to as the frequency-equivalent population, can be found by varying the population size and number of alleles of the NIL model until the frequency distribution has the same mean and variance as the DL model.

Let the DL population size and number of alleles be $N$ and $k$ and let the frequency-equivalent NIL model values be $N_{\text {fe }}$ and $k_{\text {fe }}$, respectively. Equating the mean and steady state variances of the distributions (eqn 2):

$\frac{2 N_{\mathrm{fe}}}{k_{\mathrm{fe}}}=\frac{2 N}{k}$

$$
\begin{aligned}
\frac{2 N_{\mathrm{fe}}\left(k_{\mathrm{fe}}-2\right)}{k_{\mathrm{fe}}^{2}} \cdot \frac{1}{1-\theta_{\mathrm{fe}}^{2}}= & \frac{2 N(k-2)}{k^{2}}\left(1+\frac{d^{2} \theta^{2}}{1-(1-d+d \theta)^{2}}\right) \\
& +\frac{2 N l_{p}\left(1-l_{p}\right)}{k} \frac{d^{2} \theta^{2} L^{2}}{1-(1-d+d \theta)^{2}},
\end{aligned}
$$

where $\theta_{\mathrm{fe}}=1-\frac{k_{\mathrm{fe}}}{\left(k_{\mathrm{fe}}-1\right)\left(k_{\mathrm{fe}}-2\right)}$.

For example, the DL simulation model of Brooks et al. (1996) in which $N=3840, k=16, d=0.7$, $l_{p}=0.2$ and $l_{q}=20$ has the same allele frequency distribution as the NIL model with $N_{\mathrm{fe}}=7920$ and $k_{\mathrm{fe}}=33$. The DL model has a larger variance than the NIL model of the same population size, because of the effect of the $\mathrm{L}$ factor, and so the frequencyequivalent NIL model must have a larger population size and number of alleles to produce the same variance. This contrasts with the concept of effective population size, in which variation in plant size decreases the effective population size. In terms of the allele frequency distribution, the effect of a variation in plant size is to make the population behave as if it had a larger size and more alleles. Overlapping generations has the opposite effect.

\section{The number of alleles that can be maintained in a population}

Wright (1939) calculated the number of alleles that can be maintained in a panmictic population at equilibrium based on an allele frequency probability distribution that included mutation. He related the rate of loss of alleles (the chance of an arbitrary allele becoming extinct in the next generation) to the relative frequency of the 'subterminal class' which is the probability, $P_{s}$, that the frequency of the allele in the current generation equals one. Wright's equation was:

rate of loss of alleles $=\frac{k-3}{2(k-1)} P_{\mathrm{s}}$.

There are $k$ alleles in the population and so the expected number of extinctions in one generation is:

$\frac{k(k-3)}{2(k-1)} P_{\mathrm{s}}$

Wright (1939) equated this to the average number of new mutations occurring in a single generation to obtain the number of alleles contained in the population at equilibrium.

The time series analysis presented in our previous paper (Brooks et al., 1997) gives the probability distribution of an established allele without including the effect of mutation. However, the same principle of equating gains and losses can be applied by equating the average loss of established alleles to the average rate of occurrence of mutations that become established.

The probability distribution of an established allele is the normal distribution with a mean of $2 N / k$ and a standard deviation of $\sqrt{\operatorname{var}\left(f_{\infty}\right)}$. The approximations in the time series analysis are less valid for small frequencies and so the tail of the normal distribution may not correspond closely to the true distribution. Speculatively, however, the probability, $P_{\text {est }, s}$, of an established allele occurring only once can be taken as:

(c) The Genetical Society of Great Britain, Heredity, 79, 361-364. 


$$
\begin{aligned}
P_{\text {est }, \mathrm{s}}=\text { probability } & \left(\left(-\frac{2 N}{k}+\frac{1}{2}\right) \frac{1}{\sqrt{\operatorname{var}\left(f_{\infty}\right)}}<Z_{0}<\right. \\
& \left.\left(-\frac{2 N}{k}+\frac{3}{2}\right) \frac{1}{\sqrt{\operatorname{var}\left(f_{\infty}\right)}}\right),
\end{aligned}
$$

where $Z_{0}$ is the standard normal distribution. The expected number of extinctions of established alleles in a generation is therefore:

$\frac{k(k-3)}{2(k-1)} P_{e s t, s}$.

The expected number of mutations occurring in a generation, assuming an infinite number of possible alleles, is $2 N u$, where $u$ is the mutation rate. For equilibrium, the number of extinctions must equal the number of mutations that become established. In general, the probability of a mutant allele ultimately surviving is approximately equal to twice its selective advantage (Crow \& Kimura, 1970). The probability of an allele occurring as either of the alleles of a new plant is approximately given (Brooks et al., 1997) by:

$P(q)=2 q\left(1+\frac{1-q k}{k-3+2 q}\right)$,

where $q=$ relative frequency of the allele in the source population. The expected new relative frequency is $P(q) / 2$ and so the selective advantage of the allele is:

$\frac{1-q k}{k-3+2 q} \approx \frac{1}{k-3}$ for small $q$.

The selective advantage of the allele decreases as its relative frequency increases but the probability of it surviving is $\approx 2 /(k-3)$, so that the expected number of mutations in a generation that will become established is:

$\frac{4 N u}{k-3}$.

For low mutation rates, the calculation of the number of alleles that can be maintained in the population is fairly insensitive to changes in this probability.

Equating expected gains (eqn 11) and losses (eqn 8) of established alleles, for equilibrium:

$\frac{4 N u}{k-3}=\frac{k(k-3)}{2(k-1)} P_{\text {est }, s .}$
With overlapping generations, if $d$ is small, this equation may require modifications because an allele that is absent from several successive flowering episodes may not be extinct and may reappear, and the probability of a new allele becoming established may be different. This is a complex equation, as $P_{\text {est,s }}$ is a function of $k$ and $N$. However, it can easily be solved iteratively and Fig. 1 shows the values for $u=10^{-6}$ for the NIL, L, D and DL models, for the parameter values used in the simulations of Brooks et al. (1996) given above. The value of $k$ given by eqn 12 is the number of established alleles that can be maintained by the population and does not include recent mutations, most of which will soon become extinct. However, if the mutation rate is low, the number of such alleles will be very small. The NIL model corresponds to the situation considered by Wright (1939) and the values for this model shown in Fig. 1 are close to those obtained by Wright (and subsequently verified by simulation by, for example, Crosby, 1966). Overlapping generations (D) slightly increases the number of alleles that can be maintained, whereas variation in plant size (L) reduces this number.

Alternatively, the concept of frequency-equivalent population could be used. Because the allele frequency distribution and the ratio of mutations to established alleles is the same for the given DL population and the frequency-equivalent NIL population, both populations will have the same value for Wright's (1939) variable $P_{s}$. Suppose $N_{\mathrm{fe}}$ and $k_{\mathrm{fe}}$ for the NIL model are a solution of Wright's (1939) equation (i.e. a NIL population of size $N_{\mathrm{fe}}$ can maintain $k_{\mathrm{fe}}$ alleles), so:

$2 N_{\mathrm{fe}} u=\frac{k_{\mathrm{fe}}\left(k_{\mathrm{fe}}-3\right)}{2\left(k_{\mathrm{fe}}-1\right)} P_{\mathrm{s}}$.

If the frequency-equivalent DL population with dormancy and variation in plant size has a population size $N$ and has $k$ alleles, then using eqn 3 :

$$
\begin{aligned}
2 N u & =\frac{k\left(k_{\mathrm{fe}}-3\right)}{2\left(k_{\mathrm{fe}}-1\right)} P_{\mathrm{s}} \\
& \approx \frac{k(k-3)}{2(k-1)} P_{\mathrm{s}} \quad \text { if neither } k_{\mathrm{fe}} \text { nor } k \text { is small. }
\end{aligned}
$$

The left-hand side of this equation is the expected number of mutations in one generation of the DL population and the right-hand side is the expected number of extinctions, and so the number of alleles that can be maintained by a DL population of size $N$ is $k$. The curve for the DL model, for example, can 


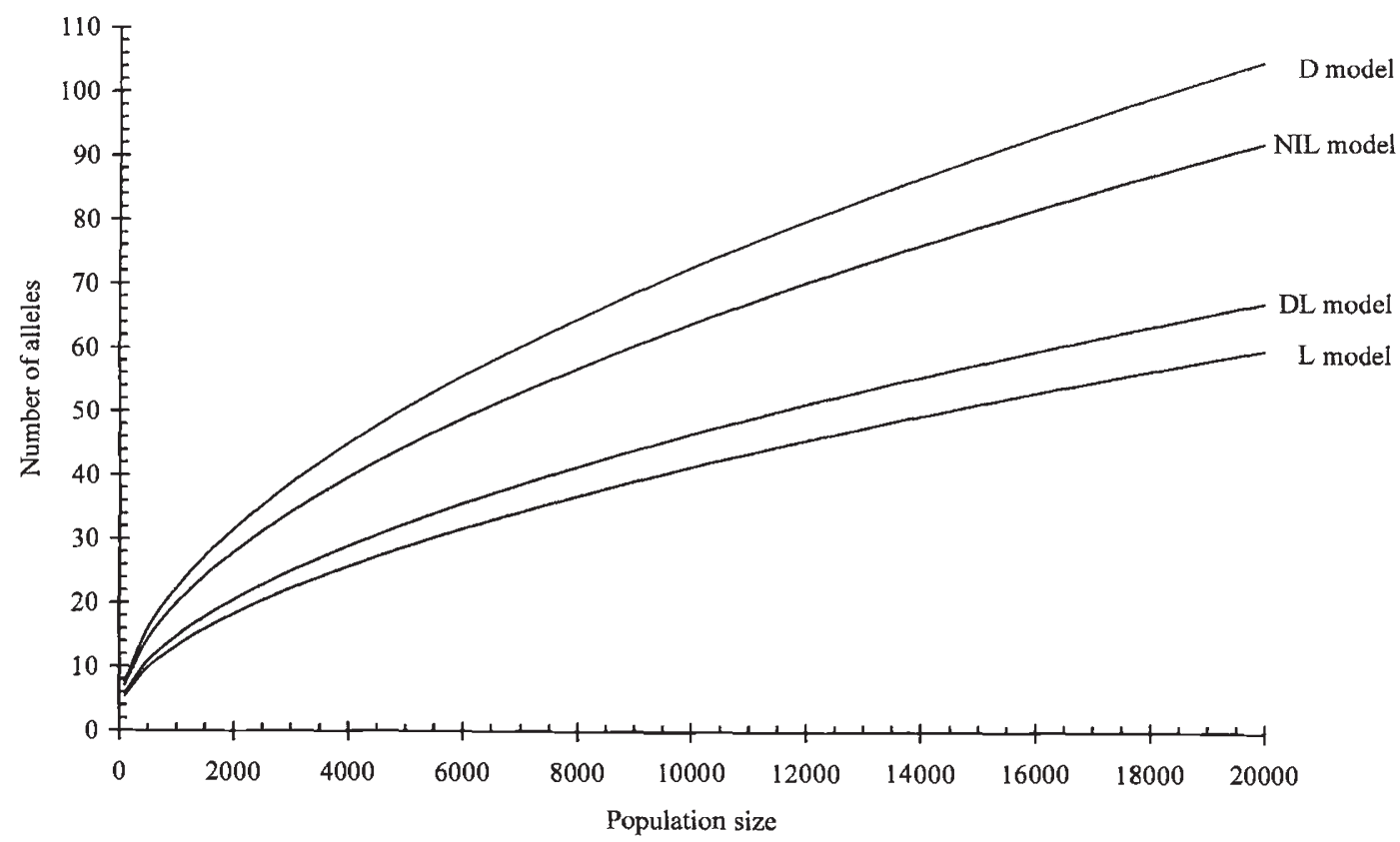

Fig. 1 The number of alleles that can be maintained in a population with an infinite number of possible alleles and a mutation rate of $10^{-6}$.

therefore be obtained by taking Wright's (1939) values for a panmictic population and, for each value of $N_{\mathrm{fe}}$ and $k_{\mathrm{fe}}$, finding the values of $N$ and $k$ for the frequency-equivalent $\mathrm{DL}$ model. This gives similar results to those obtained from eqn 12 because the results of eqn 12 for the NIL model are similar to Wright's (1939) values.

\section{Conclusion}

The time series analysis enables the number of alleles that can be maintained in a population to be estimated for populations with overlapping generations and variation in plant size. It has also been shown that there exists a panmictic population which has the same allele frequency distribution as such a population which leads to a concept of a frequencyequivalent population.

\section{References}

BROOKS, R. J., TOBIAS, A. M. AND LAWRENCE, M. J. 1996. The population genetics of the self-incompatibility polymorphism in Papaver Rhoeas. XI. The effects of limited pollen and seed dispersal, overlapping generations and variation in plant size on the variance of $S$-allele frequencies in populations at equilibrium. Heredity, 76, 367-376.

BROOKS, R. J., TOBIAS, A. M. AND LAWRENCE, M. J. 1997. A time series analysis of the population genetics of the self-incompatibility polymorphism. 1. Allele frequency distribution of a population with overlapping generations and variation in plant size. Heredity, 79, 350-360. CROSBY, J. L. 1966. Self-incompatibility alleles in the population of Oenothera organensis. Evolution, 20, 567-579. CROW, J. F. AND KIMURA, M. 1970. An Introduction to Population Genetics Theory. Harper and Row, New York. WRIGHT, s. 1939. The distribution of self-sterility alleles in populations. Genetics, 24, 538-552. 